Mens

Revue d'histoire intellectuelle et culturelle ormens

\title{
Claude Cardinal. Une histoire du RIN, Montréal, VLB éditeur,
} 2015, 512 p.

\section{Jean-Philippe Carlos}

Volume 16, numéro 2, printemps 2016

URI : https://id.erudit.org/iderudit/1041789ar

DOI : https://doi.org/10.7202/1041789ar

Aller au sommaire du numéro

Éditeur(s)

Centre de recherche en civilisation canadienne-française

ISSN

1492-8647 (imprimé)

1927-9299 (numérique)

Découvrir la revue

Citer ce compte rendu

Carlos, J.-P. (2016). Compte rendu de [Claude Cardinal. Une histoire du RIN,

Montréal, VLB éditeur, 2015, 512 p.] Mens, 16(2), 110-116.

https://doi.org/10.7202/1041789ar d'utilisation que vous pouvez consulter en ligne.

https://apropos.erudit.org/fr/usagers/politique-dutilisation/ 
S'il faut absolument saluer la recherche de pointe effectuée en amont, il faut néanmoins reconnaître que la volonté de construire un récit du voyage à Saint-Louis des premières femmes journalistes au Canada prend le dessus sur la voix des protagonistes elles-mêmes. On regrette que les témoignages ou les articles laissés par les journalistes et patiemment extirpés du silence par la chercheuse, qui restitueraient véritablement les sensibilités et les usages de l'époque, ne soient pas davantage donnés à lire. En définitive, on sait très peu de choses sur les articles issus du voyage à Saint-Louis, sur leur forme (lettre, chronique ou reportage) et sur ce qui les distingue, le cas échéant, de la production antérieure de ces femmes journalistes. En outre, la reconstitution historique ne rend pas toujours justice au travail de longue haleine mené dans les archives. L'objectif de recréer des épisodes du périple est tout à fait louable, mais il peut parfois mener à une surabondance de précisions. Aussi, la visée scientifique de l'ouvrage paraît-elle difficilement compatible avec les incursions dans les pensées intimes des journalistes, comme c'est le cas dans ces passages : "Songeant à ce qu' elle écrirait en organisant ses idées dans sa tête, Antoinette Gérin-Lajoie avait déjà décidé... " (p. 135) et "Elle semblait perdue dans ses pensées ce jour-là » (p. 124). Mises à part ces réserves, l'ouvrage de Kay est sans contredit une contribution importante à l'histoire des pratiques associatives qui ont façonné la profession de femme journaliste.

- Mylène Bédard

Département de littérature, théâtre et cinéma

Université Laval

\section{Claude Cardinal. Une histoire du RIN, Montréal, VLB éditeur, 2015, $512 \mathrm{p}$.}

Le Rassemblement pour l'indépendance nationale (RIN) est le premier grand parti politique québécois à s'être engagé à défendre l'idée que le Québec devrait être une nation souveraine et indépendante. Précurseur à de nombreux égards, le RIN est notamment 
l'un des premiers partis à s'être doté de structures démocratiques qui mettaient en valeur le rôle des militants, à avoir défendu le mode de scrutin proportionnel ainsi qu'à avoir inclus des femmes dans ses structures décisionnelles. Ancré dans une dimension internationaliste, le discours riniste était particulièrement influencé par les mouvements de décolonisation d'outre-mer, à l'instar d'un grand nombre de regroupements d'intellectuels des années 1960. Formé en grande majorité de jeunes Montréalais âgés de moins de trentecinq ans, le RIN aura également constitué une école de formation politique où de nombreux individus y auront développé une conscience politique. De par l'importance historique que revêt la place du RIN dans l'espace politique québécois, nous pouvons donc affirmer que l'étude de Claude Cardinal vient combler une lacune importante dans l'historiographie québécoise.

L'un des éléments les plus intéressants d'Une histoire du RIN est le fait que son auteur est un ancien militant du RIN. Avocat de formation, Cardinal a rejoint les rangs du parti en juin 1964 alors qu'il était âgé de seize ans. Militant de l'ombre, il a néanmoins participé à de nombreuses manifestations et il a également vécu « de l'intérieur " l'histoire du parti jusqu'à sa dissolution en 1968. Son statut d'ancien riniste permet ainsi à Cardinal de bien connaitre les tendances qui ont structuré l'existence du RIN. Qui plus est, la perspective d'un militant permet parfois d'aborder sous un angle différent les dynamiques internes d'un parti en donnant une voix aux acteurs de l'ombre. Toutefois, Cardinal s'est surtout intéressé à l'histoire du RIN en tant qu' institution, et son intérêt s'est surtout porté sur les figures dominantes du parti. Ce choix ne constitue cependant pas un défaut majeur, car le récit que nous livre l'auteur est fort captivant.

L'étude de Cardinal comporte 21 chapitres et prend la forme d'une biographie institutionnelle. Suivant une trame chronologique, l'auteur reconstruit les différentes phases d'existence du RIN, à partir de sa fondation en septembre 1960 jusqu'à son sabordement à l'été 1968. Les sources utilisées proviennent essentiellement des archives 
institutionnelles du RIN (procès-verbaux, rapports financiers, lettres de militants, journaux, etc.). Toutefois, une des lacunes de l'ouvrage apparaît dès les premières pages, puisque l'on constate qu'il n'y a pas d'introduction au sens strict du terme ni d'indications à propos de la méthodologie, du cadre conceptuel et de la problématique de recherche. Le fait que Cardinal ne soit pas un historien de formation peut expliquer ce fait, mais il est toutefois dommage que l'on entre directement dans le vif du sujet sans avoir eu droit à une certaine mise en contexte historique et méthodologique.

Les deux premiers chapitres portent sur la fondation du RIN en 1960. Mis sur pied par une vingtaine d'individus dont plusieurs étaient issus de l'Alliance laurentienne, le RIN était à l'origine un mouvement de pression destiné à sensibiliser la population du Québec à l'idéal indépendantiste. Organisation modeste, le RIN accroît néanmoins ses effectifs rapidement et obtient un certain succès auprès de la jeunesse québécoise de l'époque, qui semble réceptive au discours riniste. Celui-ci est notamment influencé par les théories anticolonialistes des intellectuels de la décolonisation africaine (Frantz Fanon, Albert Memmi, Aimé Césaire) et tend à emprunter des avenues idéologiques très progressistes, voire socialistes.

Ensuite, les chapitres 3 à 6 portent sur l'époque où le RIN était un mouvement de pression, soit de 1960 à 1963. André d'Allemagne (1960-1961) et Marcel Chaput (1961-1962), deux anciens membres de l'Alliance laurentienne, seront successivement présidents de l'organisation. Cardinal consacre d'ailleurs plusieurs pages au parcours de Marcel Chaput, un personnage pour qui il éprouve beaucoup de sympathie et dont la lecture de l'ouvrage Pourquoi je suis séparatiste (1961) aurait constitué pour lui un "appel aux armes». On apprend également que les premières années du RIN étaient difficiles sur le plan financier, comme en témoignent les états financiers, dévoilés lors du congrès national de 1962, qui accusent un déficit de plus de 4000 \$. Cette précarité n'empêche toutefois pas l'organisation de se doter d'un logo, le bélier, qui symbolise l'esprit fonceur des premiers militants rinistes, ainsi que d'un journal officiel. Dès cette époque, 
le RIN apparaît comme une formation d'avant-garde, notamment avec ses positions tranchées en faveur de l'indépendance du Québec et sa volonté de se définir comme un regroupement laïque et dépouillé de toute étiquette religieuse. Il est à noter que les querelles idéologiques font également leur apparition dès les débuts de l'aventure riniste. L'épisode le plus connu de cette période est celui du départ fracassant de Marcel Chaput en 1962, qui survient alors que ce dernier souhaite voir le RIN se transformer en parti politique. Dans la foulée, Chaput fondera le Parti républicain du Québec (PRQ) en décembre 1962, un événement qui incitera les dirigeants rinistes à accélérer leur réflexion quant au statut organisationnel de leur regroupement.

Les chapitres 7 à 13 sont consacrés à la transformation du RIN en parti politique et à la présence de l'organisation aux élections provinciales de 1966. Sous la présidence de l'avocat Guy Pouliot (1962-1964), le nouveau parti politique doit notamment se distancer des actions du Front de libération du Québec (FLQ) et des mouvements radicaux de gauche, afin de pouvoir espérer agrandir le spectre de son influence dans la population. Durant cette période, l'indépendantisme peine à s'ancrer dans le paysage politique québécois, comme en témoignent la disparition du PRQ en janvier 1964 ainsi que les nombreux cas de censure et de suspension professionnelle qui affectent plusieurs membres militants du RIN. C'est également à cette époque que Pierre Bourgault émerge en tant que figure dominante du parti, lui qui deviendra président de la formation politique en 1964. Sous sa gouverne, le parti approfondit ses réflexions concernant ses positions économiques et sociales, notamment en revendiquant un interventionnisme étatique accru, une planification rigoureuse de l'économie et en affichant une sensibilité assumée envers un idéal technocratique. Toutefois, ces orientations déplaisent franchement à la section de l'Est-du-Québec du parti, et une frange réunie autour de Jean Garon et René Jutras se dissociera du RIN pour fonder le Regroupement national (RN) en septembre 1964. Malgré tout, le parti dirigé par Bourgault se prépare au combat électoral et présente 73 candidats aux élections provinciales de 1966. Les résultats seront 
plutôt décevants puisqu'aucun candidat ne réussira à se faire élire, et le parti ne recueillera qu'à peine $5 \%$ des voix.

Les chapitres 14 à 21 sont consacrés aux dernières années du RIN, de 1966 à 1968. L'émergence d'Andrée Ferretti, une militante associée à la gauche radicale du parti, provoque des frictions idéologiques croissantes avec la frange réunie autour de Pierre Bourgault, qui exècre les positions socialistes de Ferretti. Cette querelle accaparera une bonne part des discussions politiques du parti durant cette période. La visite du général Charles de Gaulle, à l'été 1967, créera un vent d'optimisme au sein des troupes rinistes, qui sera toutefois de courte durée en raison des frictions entourant la candidature de Ferretti au poste de vice-présidente du Conseil national du RIN au congrès de 1967. La démission de Bourgault, en guise de représailles à cette candidature, engendre une crise au sein du parti et montre l'ampleur de la scission qui divise les deux camps. Malgré tout, Bourgault sera réélu président et Ferretti accédera au poste de viceprésidente, ce qui n’apaisera pas pour autant l'antipathie quauront les deux dirigeants l'un envers l'autre. La mise sur pied du Mouvement souveraineté-association (MSA) par René Lévesque, à la fin de l'année 1967, sera également une cause de frictions entre les deux franges idéologiques du RIN. Alors que Bourgault souhaite la fusion des forces indépendantistes, Ferretti prône plutôt la formule contraire afin d'éviter d'étioler le programme riniste. Les discussions entourant la possible fusion seront toutefois de courte durée, en partie à cause de la position du RIN en faveur de l'unilinguisme francophone et de la mentalité de Lévesque, qui a en horreur les méthodes de provocation rinistes. Constatant que le mouvement de René Lévesque risque d'attirer beaucoup de militants indépendantistes, dont plusieurs issus du RIN, Bourgault décide de soumettre au vote la question du sabordement du parti au congrès de 1968. Accepté par la majorité des membres, le RIN se dissout afin de privilégier l'union des forces indépendantistes et de donner la chance à cette option de s'ancrer véritablement dans le paysage politique québécois. Le livre se termine par une très courte conclusion de quatre pages, qui prend la forme 
d'un résumé un peu simpliste. Selon nous, Cardinal aurait dû porter une attention particulière à cette partie de l'ouvrage, qui ne rend compte que des caractéristiques essentielles du RIN sans tenter de resituer l'expérience riniste dans le contexte historique du Québec. L'auteur aurait ainsi pu rendre justice au reste de son livre en présentant une conclusion plus audacieuse et ouvrant sur de nouvelles perspectives.

Bien qu'il soit très bien détaillé, l'ouvrage de Cardinal souffre néanmoins de certaines lacunes qui pourront gêner les lecteurs plus avertis. Par exemple, l'auteur n'aborde que de manière très sommaire son propre parcours et son engagement au sein du RIN. Il aurait pourtant été intéressant que Cardinal recrée le contexte dans lequel il a évolué et la manière dont interagissaient les militants entre eux ainsi qu'avec les structures dirigeantes du parti. L'auteur aurait pu recentrer l'expérience riniste dans le contexte international des luttes anticolonialistes des années 1960. Cela lui aurait permis de mettre en perspective certains détails relatifs à l'idéologie riniste ainsi qu'au fonctionnement et à l'organisation du parti. Plus encore, il aurait pu souligner les liens entretenus par certains rinistes avec des membres de différents regroupements québécois, affiliés autant à la gauche qu'à la droite du spectre politique, afin de montrer que le RIN constituait en fait une coalition d'indépendantistes et non un simple regroupement d'individus de tendance gauchiste. Enfin, une analyse des liens et des similarités entre les structures et les idéologies du PQ et du RIN aurait également pu constituer une conclusion éclairante permettant de situer l'influence historique du Rassemblement dans l'histoire politique du Québec. À ce propos, Cardinal n'insiste guère sur l'influence qu'ont exercée les anciens rinistes au sein du PQ.

Malgré ces quelques réserves, l'ouvrage de Cardinal constitue néanmoins un jalon important de l'historiographie portant sur l'indépendantisme québécois. Alors que de nombreuses études avaient déjà été réalisées à propos du RIN, Cardinal a su mettre toutes les pièces du casse-tête ensemble afin de réaliser une biographie institutionnelle qui fera école dans les années à venir. Il est maintenant clair que tous 
les étudiants et les chercheurs intéressés par la question de l'indépendantisme québécoise auront désormais une synthèse historique à portée de main afin de comprendre toute la complexité et la richesse de ce pan de l'histoire politique du Québec.

- Jean-Philippe Carlos

Département d'histoire

Université de Sherbrooke

Jean-Pierre Proulx. La genèse de l'école publique et de la démocratie scolaire au Québec : les écoles de syndics 18141838, avec la collaboration de Christian Dessureault et Paul Aubin, Québec, Les Presses de l'Université Laval, 2014, 504 p.

Jean-Pierre Proulx est bien connu pour ses interventions publiques dans le domaine de l'éducation, comme journaliste, professeur à la Faculté d'éducation de l'Université de Montréal et président du groupe de travail ministériel sur la place de la religion à l'école et du Conseil supérieur de l'éducation. Cette fois-ci, il intervient, avec la collaboration de Christian Dessureault et Paul Aubin, à titre d'historien pour examiner l'histoire des écoles de syndics et reconstituer ainsi la genèse de l'école publique et de la démocratie scolaire au Québec. Une histoire méconnue, présentée le plus souvent par l'historiographie comme un épisode raté pour mettre en place la première école publique au Québec.

Jean-Pierre Proulx nous montre, par l'étude minutieuse d'un corpus de sources primaires diversifiées, que la réalité fut beaucoup plus complexe. Malgré une vie très courte - à peine sept ans, de 1829 à 1836 - les écoles de syndics, en raison de leur caractère démocratique et égalitaire, ont constitué la première tentative pour instaurer une école publique et favoriser la participation populaire à la "chose " éducative. Selon l'auteur, l'école de syndics apparaîtrait comme l'ancêtre de l'école publique québécoise, ne serait-ce que par les valeurs 